\title{
Factorization of the linear differential operator
}

\author{
Klara R Janglajew ${ }^{1 *}$ and Kim G Valeev ${ }^{2}$
}

"Correspondence:

jang@math.uwb.edu.pl

${ }^{1}$ Institute of Mathematics, University

of Białystok, Białystok, Poland

Full list of author information is

available at the end of the article

\begin{abstract}
The paper deals with the problem of factorization of a linear differential operator with matrix-valued coefficients into a product of lower order operators of the same type. Necessary and sufficient conditions for the factorization of the considered operator are given. These conditions are obtained by using the integral manifolds approach. Some consequences of the obtained results are also considered.
\end{abstract}

MSC: 34A30; 47A50; 47E05

Keywords: linear differential equations; differential operator; factorization; integral manifold of solutions

\section{Introduction}

Factorization of differential and difference operators uses analogies between these operators and algebraic polynomials. There is a number of important papers on this subject, of which we only mention a few: [1-5].

A linear differential (difference) operator $L$ admits factorization if it can be represented as a product of lower order operators of the same type (see [6-8]). Methods of factorization are exploited in analytic and algebraic approaches to the problem of integration of ordinary differential equations. Many special results are scattered over a large number of research papers; see, for instance, [9-12] and the references given therein.

In this paper, we focus on an $n$th order linear differential operator of the form

$$
L_{n}(t, D):=I D^{n}+\sum_{k=0}^{n-1} A_{k}(t) D^{k}, \quad D^{k}:=\frac{d^{k}}{d t^{k}}
$$

where we assume that $A_{k}(t)(k=0,1, \ldots, n-1)$ are $m \times m$ real-valued matrices with the entries being continuous and bounded functions on $\mathbb{R}$ and that $I$ is the $m \times m$ identity matrix.

Our purpose here is to give a proof that (1) can be presented as

$$
L_{n}(t, D)=L_{p}(t, D) L_{q}(t, D)
$$

where $p+q=n$ and

$$
L_{p}(t, D)=I D^{p}+\sum_{k=0}^{p-1} B_{k}(t) D^{k}, \quad L_{q}(t, D)=I D^{q}+\sum_{k=0}^{q-1} C_{k}(t) D^{k} .
$$

(c) 2013 Janglajew and Valeev; licensee Springer. This is an Open Access article distributed under the terms of the Creative Commons Attribution License (http://creativecommons.org/licenses/by/2.0), which permits unrestricted use, distribution, and reproduction in any medium, provided the original work is properly cited. 
We give the necessary and sufficient conditions for factorization of the above operator $L_{n}$ into the product of lower order factors $L_{q}$ and $L_{p}$. These conditions are connected with the existence of solutions of linear vector differential equations. The results are obtained by the usage of integral manifolds approach in the form elaborated by Valeev in the work [13].

\section{Splitting equations}

Let us consider the linear differential equation of order $n$, formed by acting the operator (1) on a vector function $Z$ :

$$
L_{n}(t, D) Z(t)=\left(I D^{n}+\sum_{k=0}^{n-1} A_{k}(t) D^{k}\right) Z(t)=0,
$$

where $Z(t) \in \mathbb{R}^{m}$ for $t \in \mathbb{R}$.

The linear vector differential equation (3) can be written as an equivalent system of firstorder equations of dimension $m n$. We let

$$
X(t):=\left(\begin{array}{c}
Z(t) \\
D Z(t) \\
\cdots \\
D^{q-1} Z(t)
\end{array}\right), \quad Y(t):=\left(\begin{array}{c}
D^{q} Z(t) \\
D^{q+1} Z(t) \\
\cdots \\
D^{n-1} Z(t)
\end{array}\right) .
$$

Hence,

$$
\begin{aligned}
\left(\begin{array}{c}
D Z(t) \\
D^{2} Z(t) \\
\cdots \\
D^{q} Z(t)
\end{array}\right) & \left(\begin{array}{ccccc}
0 & I & 0 & \cdots & 0 \\
0 & 0 & I & \cdots & 0 \\
\cdots & \cdots & \cdots & \cdots & \\
0 & 0 & 0 & \cdots & I \\
0 & 0 & 0 & \cdots & 0
\end{array}\right) X(t) \\
& +\left(\begin{array}{cccc}
0 & 0 & \cdots & 0 \\
0 & 0 & \cdots & 0 \\
\cdots & \cdots & \cdots & \\
0 & 0 & \cdots & 0 \\
I & 0 & \cdots & 0
\end{array}\right) Y(t)
\end{aligned}
$$

and

$$
\begin{aligned}
\left(\begin{array}{c}
D^{q+1} Z(t) \\
D^{q+2} Z(t) \\
\cdots \\
D^{n} Z(t)
\end{array}\right) & \left(\begin{array}{cccc}
0 & 0 & \cdots & 0 \\
0 & 0 & \cdots & 0 \\
\cdots & \cdots & \cdots & \cdots \\
0 & 0 & \cdots & 0 \\
-A_{0}(t) & -A_{1}(t) & \cdots & -A_{q-1}(t)
\end{array}\right) X(t) \\
& +\left(\begin{array}{ccccc}
0 & I & 0 & \cdots & 0 \\
0 & 0 & I & \cdots & 0 \\
\cdots & \cdots & \cdots & \cdots & \cdots \\
0 & 0 & 0 & \cdots & I \\
-A_{q}(t) & -A_{q+1}(t) & -A_{q+2}(t) & \cdots & -A_{n-1}(t)
\end{array}\right) Y(t) .
\end{aligned}
$$


In vector notation, we get that

$$
\frac{d X(t)}{d t}=A_{11} X(t)+A_{12} Y(t), \quad \frac{d Y(t)}{d t}=A_{21}(t) X(t)+A_{22}(t) Y(t),
$$

where $X$ is a $q m \times 1$ vector function, $Y$ is a $p m \times 1$ vector function and

$$
\begin{aligned}
& A_{11}=\left(\begin{array}{ccccc}
0 & I & 0 & \cdots & 0 \\
0 & 0 & I & \cdots & 0 \\
\cdots & \cdots & \cdots & \cdots & \\
0 & 0 & 0 & \cdots & I \\
0 & 0 & 0 & \cdots & 0
\end{array}\right) \\
& A_{12}=\left(\begin{array}{cccc}
0 & 0 & \cdots & 0 \\
0 & 0 & \cdots & 0 \\
\cdots & \cdots & \cdots & \\
0 & 0 & \cdots & 0 \\
I & 0 & \cdots & 0
\end{array}\right) \\
& A_{21}(t)=\left(\begin{array}{cccc}
0 & 0 & \cdots & 0 \\
0 & 0 & \cdots & 0 \\
\cdots & \cdots & \cdots & \cdots \\
0 & 0 & \cdots & 0 \\
-A_{0}(t) & -A_{1}(t) & \cdots & -A_{q-1}(t)
\end{array}\right) \\
& A_{22}(t)=\left(\begin{array}{ccccc}
0 & I & 0 & \cdots & 0 \\
0 & 0 & I & \cdots & 0 \\
\ldots & \ldots & \ldots & \ldots & \ldots \\
0 & 0 & 0 & \ldots & I \\
-A_{q}(t) & -A_{q+1}(t) & -A_{q+2}(t) & \cdots & -A_{n-1}(t)
\end{array}\right) .
\end{aligned}
$$

Note that the block matrices $A_{11}, A_{12}, A_{21}(t), A_{22}(t)$ are $q m \times q m, q m \times p m, p m \times q m$, $p m \times p m$ matrices, respectively.

We recall here the following definition (see [13]).

Definition 2.1 The connected subset $M$ of $\mathbb{R}^{m n+1}$ is called the integral manifold of system (4) if $\left(t_{0}, X_{0}, Y_{0}\right) \in M$ implies $(t, X(t), Y(t)) \in M$ for all $t \in \mathbb{R}$, where $X(t)$ and $Y(t)$ are determined by (4) with $X\left(t_{0}\right)=X_{0}, Y\left(t_{0}\right)=Y_{0}$.

Suppose that the linear system of differential equations (4) has an integral manifold defined by the vector equation of the form

$$
Y(t)=K(t) X(t)
$$

where $K(t)$ is a $p m \times q m$ real-valued matrix and $X, Y$ satisfy (4) on $\mathbb{R}$, i.e., provided that (6) is satisfied for a certain $t_{0} \in \mathbb{R}$, then it is valid for all $t \in \mathbb{R}$.

By differentiation of (6) with respect to $t$, we get the vector differential equation of the form

$$
\frac{d Y(t)}{d t}=\frac{d K(t)}{d t} X(t)+K(t) \frac{d X(t)}{d t} .
$$


From (4) and (7) it follows that

$$
A_{21}(t) X(t)+A_{22}(t) Y(t)=\frac{d K(t)}{d t} X(t)+K(t)\left[A_{11} X(t)+A_{12} Y(t)\right]
$$

or, equivalently,

$$
A_{21}(t)+A_{22}(t) K(t)=\frac{d K(t)}{d t}+K(t)\left[A_{11}+A_{12} K(t)\right]
$$

Let us note, that the matrix equation (8) is a Riccati-type matrix differential equation (see, for instance, [14]) and defines the integral manifold of the form (6). Assuming that the integral manifold of the form (6) does exist, the linear system (4) then reduces to

$$
\frac{d X(t)}{d t}=\left[A_{11}+A_{12} K(t)\right] X(t), \quad Y(t)=K(t) X(t)
$$

i.e., the linear subsystem splits off from the linear system of differential equations (4).

We shall seek for an integral manifold in the form (6), where $K(t)$ is a $p m \times q m$ block matrix

$$
K(t)=\left(\begin{array}{cccc}
K_{11}(t) & K_{12}(t) & \cdots & K_{1 q}(t) \\
K_{21}(t) & K_{22}(t) & \cdots & K_{2 q}(t) \\
\cdots & \cdots & \cdots & \cdots \\
K_{p 1}(t) & K_{p 2}(t) & \cdots & K_{p q}(t)
\end{array}\right) .
$$

Substituting the matrices $K(t), A_{11}$ and $A_{12}$ into (8), we obtain

$$
\begin{aligned}
& \frac{d K_{11}(t)}{d t}+K_{1 q}(t) K_{11}(t)=K_{21}(t), \\
& \frac{d K_{21}(t)}{d t}+K_{2 q}(t) K_{11}(t)=K_{31}(t), \\
& \frac{d K_{31}(t)}{d t}+K_{3 q}(t) K_{11}(t)=K_{41}(t), \\
& \ldots, \\
& \frac{d K_{p-1,1}(t)}{d t}+K_{p-1, q}(t) K_{11}(t)=K_{p 1}(t), \\
& \frac{d K_{12}(t)}{d t}+K_{11}(t)+K_{1 q}(t) K_{12}(t)=K_{22}(t), \\
& \frac{d K_{22}(t)}{d t}+K_{21}(t)+K_{2 q}(t) K_{12}(t)=K_{32}(t), \\
& \frac{d K_{32}(t)}{d t}+K_{31}(t)+K_{3 q}(t) K_{12}(t)=K_{42}(t), \\
& \ldots, \\
& \frac{d K_{p-1,2}(t)}{d t}+K_{p-1,1}(t)+K_{p-1, q}(t) K_{12}(t)=K_{p 2}(t), \\
& \frac{d K_{13}(t)}{d t}+K_{12}(t)+K_{1 q}(t) K_{13}(t)=K_{23}(t),
\end{aligned}
$$




$$
\begin{aligned}
& \frac{d K_{23}(t)}{d t}+K_{22}(t)+K_{2 q}(t) K_{13}(t)=K_{33}(t), \\
& \frac{d K_{33}(t)}{d t}+K_{32}(t)+K_{3 q}(t) K_{13}(t)=K_{43}(t), \\
& \ldots, \\
& \frac{d K_{p-1,3}(t)}{d t}+K_{p-1,2}(t)+K_{p-1, q}(t) K_{13}(t)=K_{p 3}(t),
\end{aligned}
$$

We continue in this manner to obtain

$$
\begin{aligned}
& \frac{d K_{1 q}(t)}{d t}+K_{1, q-1}(t)+K_{1 q}(t) K_{1 q}(t)=K_{2 q}(t), \\
& \cdots, \\
& \frac{d K_{p-1, q}(t)}{d t}+K_{p-1, q-1}(t)+K_{p-1, q}(t) K_{1 q}(t)=K_{p q}(t),
\end{aligned}
$$

or, in a compact form,

$$
\begin{aligned}
& \frac{d K_{s 1}(t)}{d t}+K_{s q}(t) K_{11}(t)=K_{s+1,1}(t), \\
& \frac{d K_{s j}(t)}{d t}+K_{s q}(t) K_{1 j}(t)+K_{s, j-1}(t)=K_{s+1, j}(t),
\end{aligned}
$$

where $K_{s j}(t)(s=1, \ldots, p-1 ; j=2, \ldots, q)$ are $m \times m$ matrices.

Substituting $A_{21}(t)$ and $A_{22}(t)$ into (8), we have

$$
\begin{aligned}
& \frac{d K_{p 1}(t)}{d t}+K_{p q}(t) K_{11}(t)=-A_{0}(t)-\sum_{i=1}^{p} A_{q+i-1}(t) K_{i, 1}(t) \\
& \frac{d K_{p j}(t)}{d t}+K_{p q}(t) K_{1 j}(t)+K_{p, j-1}(t)=-A_{j-1}(t)-\sum_{i=1}^{p} A_{q+i-1}(t) K_{i, j}(t),
\end{aligned}
$$

where $j=2, \ldots, q$.

Equations (10)-(13) are called the splitting equations (see [13]). We can use them for construction of an integral manifold of the linear vector differential equation (3).

The following theorem establishes the existence of an integral manifold of the linear differential equation (3).

Theorem 2.2 If there exists a solution of the system of splitting equations (10)-(13), then the linear vector differential equation (3) possesses the integral manifold of dimension mq, given by

$$
D^{q} Z(t)-\sum_{i=0}^{q-1} K_{1, i+1}(t) D^{i} Z(t)=0
$$

for $t \in \mathbb{R}$. 
Proof By virtue of formulas (5), system (9) takes the form

$$
\begin{aligned}
& D^{q} Z(t)=\sum_{i=0}^{q-1} K_{1, i+1}(t) D^{i} Z(t), \\
& D^{s+q-1} Z(t)=\sum_{i=0}^{q-1} K_{s, i+1}(t) D^{i} Z(t),
\end{aligned}
$$

where $s=2, \ldots, p$.

Differentiating each of the equations in (16) with respect to $t$, we get

$$
D^{s+q} Z(t)=\sum_{i=0}^{q-1}\left[K_{s, i+1}^{\prime}(t) D^{i} Z(t)+K_{s, i+1}(t) D^{i+1} Z(t)\right]
$$

for $s=2, \ldots, p-1$. Next, eliminating $D^{q} Z(t)$ by (15) and taking into account (10) and (11), we get that

$$
D^{s+q} Z(t)=\sum_{i=0}^{q-1} K_{s+1, i+1}(t) D^{i} Z(t)
$$

Differentiating (16) for $s=p$ and eliminating $D^{q} Z(t)$ by (15), we get, by virtue of (12)-(13),

$$
D^{n} Z(t)=-\sum_{i=0}^{q-1} A_{i}(t) D^{i} Z(t)-\sum_{i=0}^{q-1}\left(\sum_{j=1}^{p} A_{q+j-1}(t) K_{j, i+1}(t)\right) D^{i} Z(t) .
$$

Substituting into (3) the derivatives $D^{(s+q)} Z(t)(s=1, \ldots, p)$ from (17) and (18), we obtain zero. This proves the theorem.

\section{Existence of the integral manifold of solutions}

We consider the linear vector differential equation of order $q$, formed by acting $L_{q}$, given by (2), on a vector function $Z$, of the form

$$
L_{q}(t, D) Z(t)=\left(I D^{q}+\sum_{k=0}^{q-1} C_{k}(t) D^{k}\right) Z(t)=0,
$$

with a property such that each solution $Z$ of (19) is also a solution of (3) on $\mathbb{R}$. This means that $Z$ has $n$ derivatives on $\mathbb{R}$ and matrices $C_{k}(t), k=0,1, \ldots, q-1$ are differentiable up to order $p(p+q=n)$.

The following result may be proved in much the same way as Theorem 2.2.

Theorem 3.1 If any solution of the linear differential equation (19) with coefficients bounded together with their derivatives up to order $p$ satisfies (3) on $\mathbb{R}$, then the linear system of differential equations (4) has the integral manifold given by (6), where $K(t)$ is a pm $\times$ qm matrix. 
Proof Let us rewrite the linear differential equation (19) in the form

$$
D^{q} Z(t)=\sum_{i=0}^{q-1} K_{1, i+1}(t) D^{i} Z(t),
$$

where

$$
K_{1, i+1}(t)=-C_{i}(t) \quad(i=0, \ldots, q-1) .
$$

We substitute an arbitrary but fixed solution $Z$ of (20) into the differential equation (3). For this end, we differentiate (20) $p$ times with respect to $t$. After each differentiation, we eliminate $D^{q} Z(t)$ by (20) and we take into account (10)-(11). In this way we get (17). Similarly, taking into account (12)-(13), we obtain (18). Hence, the existence of solutions of the linear differential equation (19), all solutions of which are the solutions of (3), guarantees the existence of the integral manifold of the form (6) of the linear system (4) provided that all the derivatives up to the order $p$ of matrices $C_{k}(t)(k=0, \ldots, q-1)$ are bounded. This is the desired conclusion.

Remark 3.2 This kind of integral manifolds could be used in the investigation of stability of systems of difference equations (see $[15,16]$ ).

Example 3.3 Let us consider the differential equation of the fifth order

$$
L_{5}(t, D) Z(t)=\left(D^{5}+3 D^{4}+6 D^{3}+6 D^{2}+5 D+3\right) Z(t)=0
$$

and the differential equation of the third order

$$
L_{3}(t, D) Z(t)=\left(D^{3}+D^{2}+D+1\right) Z(t)=0,
$$

with a property such that each solution $Z$ of (22) is also a solution of (21). Rewrite the differential equation (22) in the form

$$
D^{3} Z(t)=-Z(t)-D Z(t)-D^{2} Z(t)
$$

Differentiating this equation two times and eliminating $D^{3} Z(t)$, we get

$$
D^{4} Z(t)=Z(t), \quad D^{5} Z(t)=D Z(t) .
$$

Indeed, substituting $D^{3} Z(t), D^{4} Z(t)$, and $D^{5} Z(t)$ from (23) and (24) into (21) yields

$$
\begin{aligned}
& D^{5} Z(t)+3 D^{4} Z(t)+6 D^{3} Z(t)+6 D^{2} Z(t)+5 D Z(t)+3 Z(t) \\
& \quad=D Z(t)+3 Z(t)+6\left(-Z(t)-D Z(t)-D^{2} Z(t)\right)+6 D^{2} Z(t)+5 D Z(t)+3 Z(t) \equiv 0 .
\end{aligned}
$$

The linear differential equation (21) can be written as

$$
\frac{d X(t)}{d t}=A_{11} X(t)+A_{12} Y(t), \quad \frac{d Y(t)}{d t}=A_{21} X(t)+A_{22} Y(t),
$$


where

$$
\begin{aligned}
& X(t):=\left(\begin{array}{c}
Z(t) \\
D Z(t) \\
D^{2} Z(t)
\end{array}\right), \quad Y(t):=\left(\begin{array}{c}
D^{3} Z(t) \\
D^{4} Z(t)
\end{array}\right), \quad A_{11}=\left(\begin{array}{ccc}
0 & 1 & 0 \\
0 & 0 & 1 \\
0 & 0 & 0
\end{array}\right), \\
& A_{12}=\left(\begin{array}{ll}
0 & 0 \\
0 & 0 \\
1 & 0
\end{array}\right), \quad A_{21}=\left(\begin{array}{ccc}
0 & 0 & 0 \\
-3 & -5 & -6
\end{array}\right), \quad A_{22}=\left(\begin{array}{cc}
0 & 1 \\
-6 & -3
\end{array}\right) \text {. }
\end{aligned}
$$

The vector equation

$$
Y(t)=\left(\begin{array}{ccc}
-1 & -1 & -1 \\
1 & 0 & 0
\end{array}\right) X(t)
$$

determines the integral manifold of the system of differential equations (25). In fact, the matrix

$$
K=\left(\begin{array}{ccc}
-1 & -1 & -1 \\
1 & 0 & 0
\end{array}\right)
$$

is a solution of the matrix equation

$$
A_{21}+A_{22} K=K\left(A_{11}+A_{12} K\right) .
$$

Thus, the existence of an integral manifold of the form (6) for the linear system (4) is equivalent to the fact that any solution of (19) satisfies (3) as well.

\section{Factorization of the operator $L_{n}(t, D)$}

Let the linear vector differential equation (3) be written in the form (4). We assume that any solution of the differential equation (19) is a solution of (3). Then the linear system (4) has the integral manifold of the form (6).

In the linear system (4), let us substitute

$$
Y(t):=K(t) X(t)+V(t)
$$

where

$$
V(t)=\left(\begin{array}{c}
V_{1}(t) \\
\cdots \\
V_{p}(t)
\end{array}\right)
$$

is a $p m \times 1$ vector for $t \in \mathbb{R}$. From the linear system (4) and (26), we obtain

$$
\begin{aligned}
\frac{d X(t)}{d t}= & \left(A_{11}+A_{12} K(t)\right) X(t)+A_{12} V(t), \\
\frac{d V(t)}{d t}= & \left(A_{21}(t)+A_{22}(t) K(t)-\frac{d K(t)}{d t}-K(t) A_{11}-K(t) A_{12} K(t)\right) X(t) \\
& +A_{22}(t) V(t)-K(t) A_{12} V(t) .
\end{aligned}
$$


Since

$$
A_{21}(t)+A_{22}(t) K(t)-\frac{d K(t)}{d t}-K(t) A_{11}-K(t) A_{12} K(t)=0,
$$

by (8), from (28) we get the splitting vector equation

$$
\frac{d V(t)}{d t}=A_{22}(t) V(t)-K(t) A_{12} V(t)
$$

which, for vectors $V_{k}(t)(k=1, \ldots, p)$, can be written in the form

$$
\begin{aligned}
& D V_{1}(t)=V_{2}(t)-K_{1, q}(t) V_{1}(t), \\
& D V_{2}(t)=V_{3}(t)-K_{2, q}(t) V_{1}(t), \\
& \ldots, \\
& D V_{p-1}(t)=V_{p}(t)-K_{p-1, q}(t) V_{1}(t)
\end{aligned}
$$

and

$$
\begin{aligned}
D V_{p}(t)= & -A_{q}(t) V_{1}(t)-A_{q+1}(t) V_{2}(t)-\cdots \\
& -A_{q+p-1}(t) V_{p}(t)-K_{p, q}(t) V_{1}(t) .
\end{aligned}
$$

From (29) we have

$$
\begin{aligned}
& V_{2}(t)=D V_{1}(t)+K_{1, q}(t) V_{1}(t) \\
& V_{3}(t)=D\left(D V_{1}(t)+K_{1, q}(t) V_{1}(t)\right)+K_{2, q}(t) V_{1}(t) \\
& V_{4}(t)=D\left[D\left(D V_{1}(t)+K_{1, q}(t) V_{1}(t)\right)+K_{2, q}(t) V_{1}(t)\right]+K_{3, q}(t) V_{1}(t)
\end{aligned}
$$

and so on. It is easily seen that

$$
V_{s}(t)=D^{s-1} V_{1}(t)+\sum_{k=1}^{s-1} D^{s-k-1} K_{k, q}(t) V_{1}(t)
$$

for $s=2, \ldots, p$. Substituting $V_{s}(t)(s=2, \ldots, p)$ into (30), we obtain the vector differential equation of order $p$

$$
\begin{aligned}
\left(D^{p}\right. & \left.+A_{n-1}(t) D^{p-1}+A_{n-2}(t) D^{p-2}+\cdots+A_{q}(t)\right) V_{1}(t) \\
& +A_{q+1}(t)\left(D V_{1}(t)+K_{1, q}(t) V_{1}(t)\right) \\
& +A_{q+2}(t)\left(D^{2} V_{1}(t)+D\left(K_{1, q}(t) V_{1}(t)\right)+K_{2, q}(t) V_{1}(t)\right)+\cdots \\
& +A_{n-1}(t)\left(D^{p-1} V_{1}(t)+D^{p-2}\left(K_{1, q}(t) V_{1}(t)\right)+D^{p-3}\left(K_{2, q}(t) V_{1}(t)\right)\right. \\
& \left.+\cdots+K_{p-1, q}(t) V_{1}(t)\right) \\
& +D^{p-1}\left(K_{1, q}(t) V_{1}(t)\right)+D^{p-2}\left(K_{2, q}(t) V_{1}(t)\right)+D^{p-3}\left(K_{3, q}(t) V_{1}(t)\right) \\
& +\cdots+K_{p, q}(t) V_{1}(t)=0 .
\end{aligned}
$$


After taking differentiation, equation (31) can be written as follows:

$$
\left(I D^{p}+\sum_{k=0}^{p-1} B_{k}(t) D^{k}\right) V_{1}(t)=0
$$

Hence, the differential equation (27) takes the form

$$
\left(I D^{q}+\sum_{k=0}^{q-1} C_{k}(t) D^{k}\right) Z(t)=V_{1}(t)
$$

where $C_{i}(t)=-K_{1, i+1}(t), i=0,1, \ldots, q-1$. Eliminating in (32) $V_{1}(t)$, we get, for the vector function $Z$, the differential equation of order $n=q+p$

$$
\left(I D^{p}+\sum_{k=0}^{p-1} B_{k}(t) D^{k}\right)\left(I D^{q}+\sum_{k=0}^{q-1} C_{k}(t) D^{k}\right) Z(t)=0
$$

or by (2)

$$
L_{p}(t, D) L_{q}(t, D) Z(t)=0
$$

for $t \in \mathbb{R}$. Now our main result follows from (33) as the next theorem.

Theorem 4.1 The linear differential operator $L_{n}(t, D)$ defined by (1) is factorized in the form

$$
L_{n}(t, D)=L_{p}(t, D) L_{q}(t, D), \quad p+q=n,
$$

where $L_{p}(t, D)$ and $L_{q}(t, D)$ are given by (2), if and only if any solution of the linear vector differential equation (19) is a solution of the linear vector differential equation (3) on $\mathbb{R}$.

\section{The case of constant coefficients}

Let us consider the case when the linear differential equation (3) has constant coefficients. This case is known to be important from the point of view of applications. In this case, from Theorem 4.1 we conclude the following.

Theorem 5.1 The polynomial matrix

$$
L_{n}(s) \equiv \sum_{k=0}^{n} A_{k} s^{k}
$$

where $A_{n}$ is an $m \times m$ nonsingular matrix, admits a factorization in the form

$$
L_{n}(s)=L_{p}(s) L_{q}(s)
$$


where

$$
\begin{aligned}
& L_{p}(s)=\sum_{k=0}^{p} B_{k} s^{k}, \quad \operatorname{det} B_{p} \neq 0, \\
& L_{q}(s)=\sum_{k=0}^{q} C_{k} s^{k}, \quad \operatorname{det} C_{q} \neq 0,
\end{aligned}
$$

if and only if any solution of the linear vector differential equation

$$
L_{q}(D) Z(t) \equiv \sum_{k=0}^{q} C_{k} D^{k} Z(t)=0
$$

is a solution of the vector differential equation

$$
L_{n}(D) Z(t) \equiv \sum_{k=0}^{n} A_{k} D^{k} Z(t)=0 .
$$

From this we have the following corollary.

\section{Corollary 5.2 The polynomial matrix}

$$
L(s) \equiv \sum_{k=0}^{n} A_{k} s^{k}, \quad \operatorname{det} A_{n} \neq 0
$$

is a multiple of sI $-C$ if and only if

$$
L(C) \equiv \sum_{k=0}^{n} A_{k} C^{k}=0
$$

Example 5.3 Consider the polynomial matrix

$$
L(s)=I s^{3}+A_{2} s^{2}+A_{1} s+A_{0} .
$$

We look for a condition under which the factorization

$$
I s^{3}+A_{2} s^{2}+A_{1} s+A_{0}=\left(I s^{2}+B_{1} s+B_{0}\right)(I s-C)
$$

is admissible. Taking the product, we get

$$
A_{2}=B_{1}-C, \quad A_{1}=B_{0}-B_{1} C, \quad A_{0}=-B_{0} C .
$$

Eliminating matrices $B_{1}, B_{0}$, we obtain the equation for the matrix $C$

$$
C^{3}+A C^{2}+A_{1} C+A_{0}=0 .
$$

On the other hand, the vector differential equation

$$
(I D-C) Y(t)=0
$$


has the fundamental matrix $Y(t)=e^{C t}$, which is a solution of the system $L(D) Y(t)=0$ under condition (34).

Remark 5.4 The main results of this paper were announced in [17].

\section{Competing interests}

The authors declare that they have no competing interests.

\section{Authors' contributions}

The authors have achieved equal contributions to each part of this paper.

\section{Author details}

${ }^{1}$ Institute of Mathematics, University of Białystok, Białystok, Poland. ${ }^{2}$ Kyiv National Economic University, Kyiv, Ukraine.

\section{Received: 6 December 2012 Accepted: 22 July 2013 Published: 7 August 2013}

\section{References}

1. Bronstein, M: On the factorization of linear ordinary differential operators. Math. Comput. Simul. 42(4-6), 386-389 (1996)

2. Coppel, WA: Disconjugacy. Lecture Notes in Mathematics, vol. 220. Springer, Berlin (1971)

3. Pòlya, G: On the mean-value theorem corresponding to a given linear homogeneous differential equation. Trans. Am. Math. Soc. 24, 312-324 (1922)

4. Zettl, A: General theory of the factorizations of ordinary linear differential operators. Trans. Am. Math. Soc. 197, 341-353 (1974)

5. Zettl, A: Explicit conditions for the factorization of $n$th order linear differential operators. Proc. Am. Math. Soc. 41(1), 137-145 (1973)

6. Etgen, GJ, Jones, GD, Taylor, WE Jr.: On the factorizations of ordinary linear differential operators. Trans. Am. Math. Soc. 297, 717-728 (1986)

7. Janglajew, K, Valeev, K: The factorization of the difference operator. Comput. Math. Appl. 42, 729-733 (2001)

8. Valeev, K, Janglajew, K: The Factorization of the Differential Expression. SVMO Preprint Series, vol. 53. Srednevolgskoye Matematicheskoye Obshchestvo, Saransk (2003)

9. Berkovich, L: Method of factorization of ordinary differential operators and some of its applications. Appl. Anal. Discrete Math. 1, 122-149 (2007)

10. Dobrogowska, A, Janglajew, K: The factorization of the $(q, h)$-difference operators. J. Differ. Equ. Appl. 13, 1171-1177 (2007)

11. Littlejohn, LL, López, JL: Variation of parameters and solutions of composite products of linear differential equations. J. Math. Anal. Appl. 369, 658-670 (2010)

12. Janglajew, KR, Valeev, KG: Conditions for factorization of linear differential-difference equations. Tatra Mt. Math. Publ. 54, 93-99 (2013)

13. Valeev, KG: Splitting of Matrix Spectra. 'Vishcha Shkola', Kiev (1986) (in Russian)

14. Abazari, R: Solution of Riccati types matrix differential equations using matrix differential transform method. J. Appl. Math. Inf. 27(5-6), 1133-1143 (2009)

15. Janglajew, K: On the reduction principle of difference equations. Dyn. Contin. Discrete Impuls. Syst. 6, 381-388 (1999)

16. Reinfelds, A, Janglajew, K: Reduction principle in the theory of stability of difference equations. Discrete Contin. Dyn. Syst. 2007, supplement, 864-874 (2007)

17. Janglajew, K: Construction of an integral manifold for linear systems. Paper presented at the Israeli-Polish mathematical meeting, PTM, Isr. Math. Union (org.), Lodz, Poland, 11-15 Sept. 2011

doi:10.1186/1687-1847-2013-237

Cite this article as: Janglajew and Valeev: Factorization of the linear differential operator. Advances in Difference Equations 2013 2013:237. 